\title{
AMQ e POA: diferenças e semelhanças na avaliação da Estratégia de Saúde da Família
}

\author{
$A M Q$ and POA: differences and similarities in the \\ Family Health Program evaluation \\ AMQ y POA: diferencias y similitudes en la evaluación \\ de la Estrategia de Salud Familiar
}

Camila Ament Giuliani dos Santos Franco ${ }^{1 *}$, Nilza Teresinha Faoro², Maria Terumi Maruyama Kami ${ }^{3}$, Ademar Cezar Volpi ${ }^{4}$

Palavras-chave: Atenção Primária à Saúde Avaliação

Saúde da Família

\section{Resumo}

Introdução: Instituída pelo Ministério da Saúde (MS), a Avaliação para Melhoria da Qualidade (AMQ) é um instrumento de avaliação e aperfeiçoamento das equipes da Estratégia de Saúde da Família (ESF). A Secretaria Municipal da Saúde de Curitiba conta, desde 2002, com o Plano Operativo Anual (POA) que é uma estratégia de gestão voltada para resultados, aprimoramento, planejamento e avaliação. Este instrumento é composto por 81 indicadores. Assim, o município do estudo conta com duas formas de avaliação de qualidade em todas as suas Unidades de Saúde da Família. 0 objetivo deste trabalho foi analisar as concordâncias e discordâncias entre 0 POA e a AMQ nos programas da saúde da criança, adolescente, mulher, diabéticos, hipertensos e saúde mental. Metodologia: Participaram do estudo 12 equipes de Saúde da Família. Para realizar a comparação, foram levantados os indicadores que estavam presentes tanto na AMQ (instrumento 5) quanto no POA-2010. Depois das comparações, foram realizadas porcentagens simples e análise das discordâncias. Resultados: No total, 28 indicadores estavam presentes tanto na AMQ como no POA. No geral houve concordância de respostas em $53,6 \%$. Na avaliação da AMQ, 82\% das respostas foram consideradas totalmente adequadas para 0 item avaliado, enquanto na avaliação realizada pelo POA este índice caiu para 46,4\%. Conclusão: Observou-se discordância entre os itens comparados da AMQ e do POA. Alguns fatores podem ser responsáveis pela discordância, entre eles: a utilização de dados recuperados automaticamente através das ações registradas pela equipe no prontuário eletrônico no POA e a obrigatoriedade da sua realização. Além disso, a realização facultativa da AMQ e a não parametrização das respostas (SIM × NÃO) podem contribuir para a divergência das respostas.

Keywords: Primary Health Care

Evaluation

Family Health

\section{Abstract}

Introduction: Quality Enhancement Evaluation (QEE) is an instrument established by the Ministry of Health (MH) in order to assess and improve Family Health Program (FHP) teams. Since 2002, the Municipal Secretary of Health of Curitiba relies on the Yearly Operating Plan (YOP), a management strategy directed to results, enhancement, planning and assessment of public health. YOP is a tool comprising 81 indicators. Thus, the city counts on two different forms of quality evaluation at all its Family Health Program units. Objective: The purpose of this article was to analyze the agreements and disagreements between QEE and YOP on the following programs of the Municipal Secretary

\footnotetext{
1 Secretaria Municipal de Saúde, Curitiba, Brasil. cafranco@sms.curitiba.pr.gov.br

${ }^{2}$ Secretaria Municipal de Saúde, Curitiba, Brasil. nifaoro@sms.curitiba.pr.gov.br

${ }^{3}$ Secretaria Municipal de Saúde, Curitiba, Brasil. mkami@sms.curitiba.pr.gov.br

${ }^{4}$ Secretaria Municipal de Saúde, Curitiba, Brasil. avolpi@sms.curitiba.pr.gov.br

${ }^{*}$ Autor correspondente.

Fonte de financiamento: nenhuma.

Conflito de interesses: declaram não haver.

Recebido em: 20/06/2011

Aprovado em: 23/01/2012
} 
of Health: Children Health, Adolescent Health, Women Health, Diabetes, Hypertensive Condition and Mental Health. Methodology: The study included 12 FHP teams. In order to carry out a comparative study, AMQ (Instrument 5) and POA (2010) indicators were researched. Simple percentage and discordance analyses were calculated after comparison. Results: QEE and YOP totalized 28 indicators. There was an overall response concordance of $53.6 \%$. Eighty-two percent of the QEE responses were considered totally adequate for the assessed item, while the POA index showed $46.4 \%$. Conclusion: Discordance was observed between the compared items of QEE and YOP. Some of the following factors can be responsible for this discordance: the use of data automatically retrieved through the actions registered by the team on the electronic medical record at YOP and the obligation of its completion. In addition, the QEE non-mandatory data recording and the non-parameterization of the (YES/NO) answers may have contributed to the divergence of the responses.

\section{Palabras clave: \\ Atención Primaria a la Salud \\ Evaluación \\ Salud de la Familia}

\section{Resumen}

Introducción: Establecido por el Ministério de Salud (MS), la Evaluación para la Mejora de Calidad (Avaliação para Melhoria da Qualidade - AMQ) es un instrumento de evaluación y mejora de los equipos de la Estratégia Salud de la Familia (ESF). La Secretaria Municipal de Salud de Curitiba cuenta, desde 2002, con el Plan Operativo Anual (POA) que es una estrategia de gestión orientada para resultados, mejora, planicicación y evaluación. Este instrumento está compuesto por 81 indicadores. De esta forma, el municipio de este estudio cuenta con dos formas de evaluación de calidad en todas sus Unidades de Salud de la Familia. El objetivo de este trabajo fue analizar los acuerdos y desacuerdos entre POA y AMQ en los programas de salud del niño, adolescente, mujer, diabéticos, hipertensos y salud mental. Metodología: Participaron del estudio 12 equipos de Salud de la Familia. Para realizar la comparación fueron levantados indicadores que estaban presentes tanto en la AMQ (instrumento 5), cuanto en el POA-2010. Después de las comparaciones fueron realizados porcentajes simples y análisis de los desacuerdos. Resultados: En total, 28 indicadores estaban presentes tanto en la AMQ como en el POA. En general hubo acuerdo de respuestas en 53,6\%. En la evaluación de la AMQ, 82\% de las respuestas fueron consideradas totalmente adecuadas para el íten evaluado, mientras en la evaluación realizada por el POA este indice cayó para 46,4\%. Conclusión: Se observó desacuerdo entre los ítens comparados de la AMQ y del POA. Algunos factores pueden ser responsables por el desacuerdo entre ellos: la utilización de datos recuperados automáticamente a través de las acciones registradas por el equipo en el registro médico electrónico en el POA y la obligatoriedad de su realización. Además, la realización facultativa de la AMQ y la no parametrización de las respuestas (SÍ × NO) pueden contribuir para la divergencia de las respuestas.

\section{Introdução}

A Atenção Primária à Saúde (APS) pode ser definida como um conjunto de ações de saúde, no âmbito individual e coletivo, que abrange a promoção e a proteção da saúde, a prevenção de agravos, o diagnóstico, o tratamento, a reabilitação e a manutençáo da saúde ${ }^{1}$.

Dentro desta ótica de atenção, Starfield ${ }^{2}$ coloca como aspectos especificamente pertinentes à prática da APS: o acesso, o tamanho do grupo acompanhado, a variedade de serviços fornecidos, a continuidade e os mecanismos para melhorar o reconhecimento das informaçóes a respeito dos pacientes.

Para garantir que estes aspectos essenciais da APS estejam presentes é necessário que ocorram avaliaçóes destes serviços e estas devem envolver, segundo Campos $^{3}$, a estrutura, o processo e o nível de qualidade alcançado, incluindo os problemas, as falhas, e a busca de estratégias para a sua correção ou melhoria.

A avaliação deve ter o objetivo de qualificar a APS, promovendo a construçáo de processos estruturados e sistemáticos, coerentes com os princípios do Sistema Único de Saúde (SUS) .

É preconizado pela Política Nacional da Atenção Básica ${ }^{1}$ que as equipes devem, durante seu processo de trabalho, acompanhar e realizar avaliaçoóes sistemáticas das ações implementadas visando à readequaçáo do seu processo. Desta forma, o Ministério da Saúde desenvolveu um conjunto de iniciativas para a avaliação e o monitoramento dos serviços de saúde 5 .

Dentro deste panorama a cidade de Curitiba, em 2002, discutiu e criou, por meio de oficinas gerenciais, os "Contratos de Gestão" que possuem dois instrumentos - Termos de Compromisso (TERCOM) e os Planos Operativos Anuais (POA).

Os contratos de gestão são firmados anualmente entre as Equipes das Unidades de Saúde (US) representadas pela Autoridade Sanitária Local (ASL), Supervisor Distrital e Secretário Municipal de Saúde e representantes do Conselho Local de Saúde.

O Ministério da Saúde implantou, em 2005, a Avaliação para Melhoria da Qualidade (AMQ) da Estratégia Saúde da Família (ESF) com o objetivo de oferecer aos gestores municipais ferramentas de avaliação e de gestão da qualidade da $\mathrm{ESF}^{7}$. Este manual foi baseado no modelo proposto por Donabedian (1980 apud Brasil, 2005) ${ }^{8}$, que “[...] considera os elementos de estrutura, de processo e de resultado, tendo como foco de análise os serviços de saúde e as práticas assistenciais." ${ }^{\circ}$.

Assim sendo, mostra um "[...] panorama da organização e do funcionamento do serviço de saúde, possibilitando a identificação dos estágios de desenvolvimento e de seus aspectos críticos, assim como das potencialidades e dos pontos 
já consolidados." de intervençôes sistematizado dos problemas encontrados na avaliação.

O município de Curitiba aderiu à AMQ em seu primeiro ano de implantação, 2005, tendo, portanto, as duas formas de avaliação estabelecidas e praticadas pelos serviços da ESF.

Neste panorama, este trabalho tem por objetivo realizar a análise das concordâncias e discordâncias entre o POA e a AMQ nos programas da saúde da criança, adolescente, mulher, diabéticos, hipertensos e saúde mental em Unidades de Saúde do município de Curitiba-PR.

\section{Metodologia}

Atualmente, a rede municipal de atenção primária à saúde conta com 109 Unidades Básicas correspondendo a 100\% da rede básica, sendo 55 Unidades com ESF totalizando 170 equipes.

A cidade está dividida em nove distritos sanitários. Para a realização deste trabalho, foram selecionadas todas as US da ESF de um distrito sanitário, contando com 12 equipes da ESF.

Para realizar a comparação, foram levantados os indicadores que estavam presentes tanto na AMQ (instrumento 5) ${ }^{8}$ quanto no POA-2010.

O POA é uma planilha anexa ao TERCOM que relaciona os indicadores de saúde priorizados para o monitoramento durante o ano ${ }^{6}$. Tem monitoramento trimestral, proporcionando às equipes o conhecimento permanente dos resultados e favorecendo a criação de estratégias de enfretamento para melhoria dos indicadores pactuados. Possui 81 indicadores de saúde priorizados para o monitoramento no ano de 2010.

A AMQ é composta por cadernos dirigidos a atores específicos: gestores, coordenadores, unidades de saúde e equipes. Através da "[...] autogestão permite que os próprios atores envolvidos com a estratégia possam avaliá-la de maneira sistêmica e integrada, com vistas ao aprimoramento contínuo do serviço de saúde." 5 . Os eixos norteadores do instrumento baseiam-se no ciclo vital (Saúde da Criança, Saúde do Adolescente, Saúde do Adulto e do Idoso) dividindo em cinco padrôes de qualidade, que são: elementar, em desenvolvimento, consolidada, boa e avançada ${ }^{8}$.

O instrumento $5 \mathrm{da} A M Q$ é dirigido às equipes Saúde da Família, especificamente aos profissionais de nível superior, devendo ser respondido pelo médico, cirurgiáo dentista e enfermeiro, e conta com 90 questóes.

Dentro do ciclo vital, optou-se por dividir em saúde da criança, saúde da mulher, saúde mental, saúde do adolescente, hipertensão e diabetes, permitindo, desta forma, analisar os itens que são avaliados igualmente na AMQ e no POA.

Como instrumentos de comparação, foram selecionadas as fichas de resposta do instrumento 5 e encaminhadas para a Secretaria da Saúde de Curitiba pelas equipes da ESF e as planilhas do POA, que contam com todas as avaliaçóes dos 81 itens no ano de 2010.

Foram analisados todos os itens que realizavam a mesma avaliação tanto na AMQ como no POA, realizando leitura comparativa para cada item.

O POA contém as respostas em porcentagem da cobertura que foi atingida. Foram atribuídos os mesmos valores para cada unidade de saúde, somando a porcentagem e dividindo pelo número de Unidades, resultando uma média geral. As avaliaçōes da AMQ foram somadas da seguinte forma: SIM representa $100 \%$ e NÂO representa $0 \%$. Somaram-se as respostas das 12 equipes e foram realizadas as médias simples.

Consideraram-se itens concordantes aqueles que foram avaliados como $100 \%$ no POA e a resposta como SIM na AMQ ou diferente de $100 \%$ no POA e NÁO na AMQ; e considerados como discordantes quando apresentavam SIM na AMQ e menor do que $100 \%$ no POA ou entáo NÁO na AMQ e $100 \%$ no POA.

Depois das comparaçóes, foram realizadas porcentagens simples e análise das discordâncias.

\section{Resultados}

No total, 28 indicadores estavam presentes tanto na AMQ como no POA. Foram divididos da seguinte forma: saúde da criança com seis itens; saúde do adolescente e saúde mental com duas questóes; saúde da mulher com oito incisos; hipertensão e diabete melito com cinco elementos.

No geral, houve concordância de respostas em 53,6\%, apresentando a mesma resposta em 15 dos 28 itens avaliados. Dentre os itens avaliados como concordantes, pode-se citar o item 5.6 do instrumento 5 da AMQ, que obteve resposta SIM em todas as avaliaçóes realizadas por todas as equipes, e o item 4.5 do POA que atingiu esta meta em $100 \%$ das equipes avaliadas. O item 5.6 do POA propóe que $90 \%$ ou mais das crianças da área com até 1 ano de vida estejam com esquema de vacinação em dia, e o item 4.5 do POA indica que cada equipe tenha no máximo $5 \%$ das crianças menores de 1 ano acompanhadas com esquema vacinal não adequado.

Entre os itens avaliados como discordantes, podemos citar o item 5.61 da AMQ, que foi avaliado como SIM por todas as equipes, e o item 10.3 do POA que evidenciou cobertura de $63 \%$ no item avaliado. O item 5.61 do AMQ sugere 
que a ESF deve manter acompanhamento do tratamento pela referência dos usuários de álcool e outras drogas, e o item 10.3 do POA propóe cobertura de acompanhamento de todos os usuários inscritos no Programa de Saúde MentalDependência Química.

O Figura 1 representa as concordâncias entre as respostas encontradas na AMQ e no POA.

$\mathrm{Na}$ avaliação da AMQ, $82 \%$ das respostas foram consideradas totalmente adequadas para o item avaliado (resposta SIM), enquanto na avaliação realizada pelo POA, este índice caiu para $46,4 \%$.

O Figura 2 apresenta as correlaçóes entre a adequaçáo da resposta na AMQ e o cumprimento das metas do POA. Dentre os itens avaliados, a AMQ foi considerada como realizando as açóes avaliadas plenamente em 5 das 6 grandes áreas avaliadas, em contrapartida o POA não apresentou avaliação de $100 \%$ em nenhuma grande área avaliada, conseguindo atingir o item estabelecido em $81 \%$ das açóes propostas em âmbito geral.

\section{Discussão}

A avaliação em saúde e, em especial, na atenção primária é fonte de discussão em muitos países do mundo. $\mathrm{O}$ modelo desenvolvido no Brasil pelo Ministério da Saúde tem

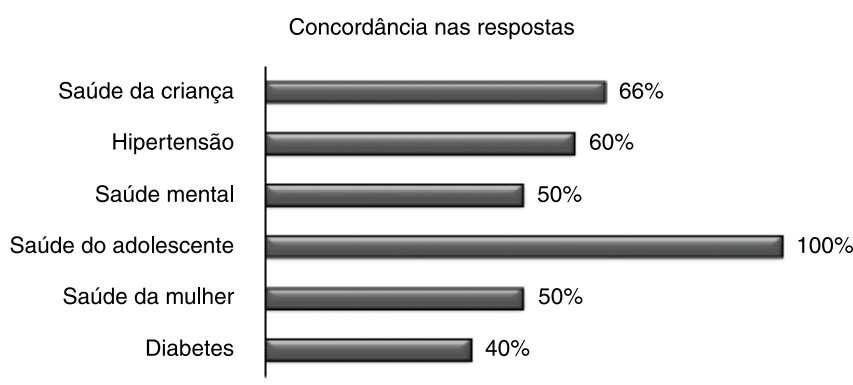

Figura 1. Comparações entre as respostas por item avaliado.

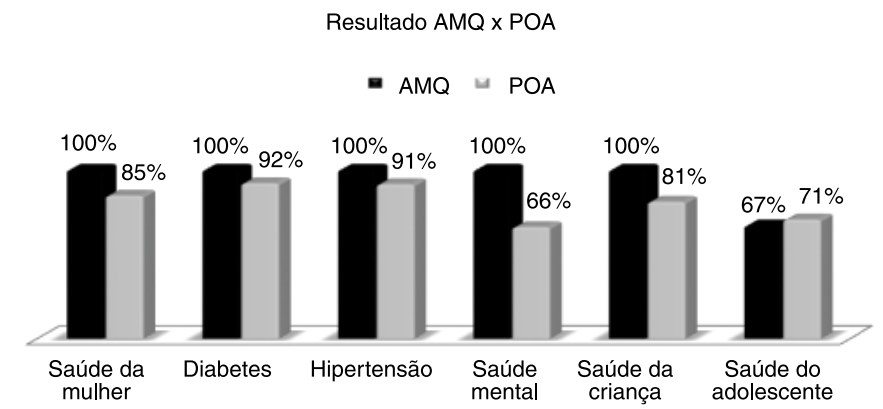

Figura 2. Resultado referente à avaliação (em \%) de cada grupo avaliado entre $A M Q$ e $P O A$. representação muito semelhante às avaliaçóes realizadas pelo governo português, tendo o mesmo objetivo no resultado das avaliaçóes: "Este momento de reflexão crítica vale, acima de tudo, pelo fato de ser plural, isto é, mobiliza e convoca a diversidade de olhares e experiências de todos os membros da equipe, sem exceção, a participarem neste processo de questionamento e de sinalização de evidências demonstradoras das práticas da equipe." 9

Ao comparar os resultados apresentados no POA e na AMQ, observou-se como uma importante dificuldade a forma de avaliação realizada pela $\mathrm{AMQ}$, na qual os atores podem responder somente SIM ou NÂO, gerando impasses para a resposta da equipe de saúde, mesmo considerando que o instrumento apresenta índice explicativo para cada pergunta.

Ao realizar comparaçôes com outros índices avaliativos como o MoniQuor ${ }^{9}$, observa-se que a resposta de forma escalonada, como no caso de $0-1$, facilita a resposta e fornece resultados mais próximos da realidade local das US.

Os resultados apresentados mostram uma grande porcentagem de respostas "SIM" que podem não refletir a real situação da equipe da ESF e sim uma não adequaçáo à resposta "NÃO", representando desta forma a melhor opção, porém não a totalmente correta.

Ao analisar itens não parametrizados, comparando escalas com respostas SIM ou NÃO, pode-se incorrer no erro da análise estatística, porém apresenta e reforça a necessidade de haver respostas intermediárias para um instrumento de avaliação em saúde.

Em contrapartida, o POA nos dá dados fidedignos extraídos do prontuário eletrônico utilizado pelo município, mas não contempla uma avaliação sistemática com planos de intervençáo como na AMQ, não tendo seus dados analisados por todos os membros da equipe necessariamente.

Em geral, as avaliaçôes realizadas utilizando a AMQ mostraram resultados mais positivos do que os do POA, podendo refletir questóes como: a pouca disponibilidade da equipe em priorizar a autoavaliaçáo e planejamento, já que esta não gera cobrança, centrando-se no cumprimento de metas e tarefas determinadas no nível central ${ }^{10}$; a visão otimista da equipe ou a dificuldade em realizar autoavaliaçôes, lembrando que os profissionais das equipes são formados em um modelo biológico cartesiano em que não são aceitas falhas.

\section{Conclusão}

Conclui-se que a utilização das duas formas de avaliação (AMQ e POA) são complementares, devendo ser trabalhadas em todos os âmbitos de gestão para que seus objetivos sejam contemplados e a melhoria da qualidade de serviço seja atingida. 


\section{Referências}

1. Brasil. Ministério da Saúde. Secretaria de Atenção à Saúde. Departamento de Atenção Básica. Política nacional de atenção básica. Brasilia: Ministério da Saúde; 2011.

2. Starfield B. Atenção Primária: equilíbrio entre necessidades de saúde, serviços e tecnologia. Brasília: UNESCO, Ministério da Saúde; 2002.

3. Campos CEA. Estratégias de avaliação e melhoria contínua da qualidade no contexto da Atenção Primária à Saúde. Rev Bras Saúde Mater Infant. 2005; 5(suppl.1): [online] [acesso em 2011 Abr. 20]. Disponível em: http://www.scielo.br/scielo.php?script=sci_ arttext\&pid=S1519-38292005000500007\&lng=en\&nrm=iso. http:// dx.doi.org/10.1590/S1519-38292005000500007

4. Felisberto E. Monitoramento e avaliação na atenção básica: novos horizontes. Rev Bras Saude Mater Infant. 2004; 4(3):[online] [acesso em 2011 Abr. 20]. Disponível em: http://www.scielo.br/scielo. php?script=sci_arttext\&pid=S1519-38292004000300012\&lng=en \&nrm=iso. http://dx.doi.org/10.1590/S1519-38292004000300012

5. Silva JM, Caldeira AP. Avaliação para melhoria da qualidade da estratégia saúde da família e a qualificação profissional.TrabEduc Saúde. 2011; 9(1):[online] [acessoem 2011 Jun. 15]. Disponívelem: http://www.scielo.br/scielo.php?script=sci_arttext\&pid=S1981- 77462011000100007\&lng=en\&nrm=iso. http://dx.doi.org/10.1590/ S1981-77462011000100007

6. Ducci, L. Curitiba firma contrato de gestão com metas para a saúde. Rev Bras Saúde Fam. 2007; 14: 48-57.

7. Brasil. Ministério da Saúde. Secretaria de Atenção à Saúde. Departamento de Atenção Básica. Avaliação para melhoria da qualidade da Estratégia Saúde da Família. Brasília: Ministério da Saúde; 2005.

8. Brasil. Ministério da Saúde. Secretaria da Atenção à Saúde. Departamento de Atenção Básica. Avaliação para melhoria da qualidade da estratégia saúde da família. Brasilia: Ministério da Saúde; 2005. 6 v. (Série B. Textos Básicos de Saúde).

9. Portugal. Ministério da Saúde. Cuidados de Saúde Primário. O processo de auto diagnóstico: metodologia e critérios do autodiagnóstico quantitativo - MoniQuor adaptado às USF - e qualitativo - fichas reservas do MoniQuor USF - na avaliação das pré-candidaturaa a modelo B [online]. Lisboa; 2007. Disponívelem:http//www.mcsp. min-saude. pt/engine. php?cat $=46$

10. Trad LAB, Rocha AARM. Condições e processo de trabalho no cotidiano do Programa Saúde da Família: coerência com princípios da humanização em saúde. Ciênc Saúde Coletiva. 2011; 16(3): [online] [acesso em 2011 Jun 16]. Disponível em: http://www.scielo.br/scielo. php?script=sci_arttext\&pid=S1413-81232011000300031\&lng=en . http://dx.doi.org/10.1590/S1413-81232011000300031 